\title{
Discursos de docentes costarricenses de secundaria sobre la dis/capacidad
}

Discourses of Costa Rican secondary school teachers about dis/ability

\author{
Volumen 21, Número 2 \\ Mayo - Agosto \\ pp. 1-27
}

Daniel Fernández Fernández

Citar este documento según modelo APA

Fernández Fernández, Daniel. (2021). Discursos de docentes costarricenses de secundaria sobre la dis/capacidad. Revista Actualidades Investigativas en Educación, 21(2), 1-27. Doi. 10.15517/aie.v21i2.46778 


\title{
Discursos de docentes costarricenses de secundaria sobre la dis/capacidad \\ Discourses of Costa Rican secondary school teachers about dis/ability
}

\section{Daniel Fernández Fernández ${ }^{1}$}

\begin{abstract}
Resumen: En este artículo se presenta parte de los resultados de una investigación desarrollada en el Instituto de investigación en Educación de la Universidad de Costa Rica que tiene como uno de sus intereses generales responder a la pregunta: ¿Cuáles son las concepciones que devela el discurso de docentes costarricense de secundaria en torno al tema de la discapacidad? Para responder a esta pregunta, a nivel metodológico, se elaboró un acercamiento de carácter cualitativo en el que se realizaron entrevistas semiestructuradas a docentes de secundaria pertenecientes al ámbito de educación pública costarricense. A partir de dicho material, se desarrolló un análisis discursivo que se basó en un procedimiento de identificación de proposiciones textuales y una elaboración de implicaciones discursivas. Los resultados muestran que la oposición normalidad - anormalidad resulta un elemento clave para comprender las construcciones discursivas del profesorado. De esta forma, se identificaron núcleos discursivos como la idea de la discapacidad como una carga y como una deficiencia, así como otro conjunto de intervenciones que fueron clasificadas como discursos críticos en torno a la diversidad funcional. Una de las principales conclusiones del artículo es que, a pesar de los importantes cambios en materia de legislación y políticas públicas en materia de discapacidad que atañen al ámbito educativo, persisten una serie de concepciones que develan visiones prejuiciadas sobre lo que se entiende por discapacidad. Una posible aplicación del presente artículo es justamente identificar las concepciones estigmatizantes que se vuelven recurrentes, $y$ tomarlas como un punto de partida para establecer un diálogo reflexivo con el profesorado.
\end{abstract}

Palabras clave: docentes, enseñanza secundaria, discurso, discapacidad.

Abstract: This article presents part of the results of a research developed at the Research Institute in Education of the University of Costa Rica that has one of its general interests to answer the question: What are the concepts that the discourse of Costa Rican secondary school teachers reveals about the issue of disability? To answer this question, a qualitative approach was developed at the methodological level, for which semi-structured interviews were conducted with secondary school teachers belonging to the Costa Rican public education sector. From this material, a discursive analysis was developed based on a procedure of identification of textual propositions and the elaboration of discursive implications. The results show that the opposition normality - abnormality is a key element to understand the teachers' discursive constructions. In this way, discursive cores are identified as the idea of disability as a burden and as an impairment, as well as another set of interventions that were classified as critical discourses around functional diversity. One of the main conclusions of the article is that, despite the important changes in legislation and public policies regarding disability that affect the educational field, there are still a number of conceptions that reveal prejudiced views about what is understood by disability. One possible application of this article is precisely to identify the stigmatizing conceptions that become recurrent and take them as a starting point to establish a reflexive dialogue with teachers.

Keyword: teachers, secondary education, discourse, disability.

${ }_{1}^{1}$ Universidad de Costa Rica, Instituto de Investigación en Educación, San José, Costa Rica. Orcid: http://orcid.org/0000-0002-8744-1890

Dirección electrónica: daniel.fernandez f@ucr.ac.cr

Artículo recibido: 16 de noviembre, 2020

Enviado a corrección: 23 de febrero, 2021

Aprobado: 19 de abril, 2021

Los contenidos de este artículo están bajo una licencia Creative Commons 


\section{Introducción}

El presente artículo forma parte de la investigación denominada Diversidad sexual y funcional: Análisis del discurso de docentes y estudiantes de secundaria, inscrita en Instituto de Investigación en Educación de la Universidad de Costa Rica. Dicho proyecto tiene como objetivo general analizar las formaciones discursivas en torno a la diversidad sexual y funcional de docentes y estudiantes de secundaria pertenecientes al ámbito de educación formal regular costarricense. El presente texto se aboca, específicamente, al análisis del discurso de docentes en lo relativo a la diversidad funcional.

El concepto de discapacidad es sin duda el que identifica con mayor facilidad todo un amplio espectro de características asociadas a la funcionalidad corporal y mental que, usualmente, a nivel social, se vuelve objeto de diferentes formas de estigmatización y prejuicio. En este sentido, concebimos que dicha noción representa un significante sociocultural que no puede ser soslayado, razón por la cual lo hemos tomado como objeto discursivo central de esta investigación, a pesar de que conceptualmente nuestro posicionamiento teórico parte de la crítica al "capacitismo". Por esta razón, se destaca el concepto de discapacidad en cursiva, para hacer la distinción de su uso como objeto estudio y no como representación del posicionamiento teórico del redactor del presente texto.

En esta dirección, se arguye que tomar el discurso de docentes de secundaria sobre discapacidad como objeto de estudio resulta una tarea de gran relevancia, puesto que, al comprender cómo opera esta articulación discursiva, podemos acercarnos a conocer cuál es el lugar que ocupa actualmente la discapacidad en el ámbito educativo, más allá del desarrollo de los avances que puedan existir actualmente en materia legal y en políticas públicas. Como señala Meléndez (2018), en Costa Rica, en lo que respecta a la protección de los derechos y la promoción de la participación en la sociedad de personas con discapacidad, el estado costarricense ha generado importantes cambios tanto a nivel de normativas jurídicas (Costa Rica, Asamblea Legislativa, 1996, 2008, 2016) como en lo que se refiere a la implementación de políticas institucionales (Consejo Nacional de Rehabilitación y Educación Especial [CNREE], 2011).

En esta misma dirección apunta Quijano (2008) cuando plantea que en Costa Rica se han realizado las acciones pertinentes para establecer los fundamentos legales que permitan la igualdad de oportunidades para el estudiantado con necesidades educativas especiales asociadas a algún tipo de discapacidad. La autora enfatiza que la Ley 7600 , denominada "Ley de Igualdad de Oportunidades para las Personas con Discapacidad", ha mejorado la inclusión 
de niños, niñas (y agregamos adolescentes) con necesidades educativas especiales a los diferentes ciclos de la Educación General Básica, al generar mejores condiciones para que sus necesidades sean atendidas en los distintos niveles del proceso de educación formal. Dichos avances alcanzados por el país son igualmente coincidentes con la tendencia general a nivel latinoamericano, que ha supuesto la suscripción de convenciones y consensos en la materia (Stang, 2011, González y Stang, 2014).

Como se sugirió anteriormente, si bien todos estos avances son imprescindibles, resulta necesario preguntarse hasta qué punto se han generado las transformaciones necesarias del tejido sociocultural que garanticen condiciones reales de equidad para las personas con discapacidad. Segato (2003) señala, refiriéndose a las distintas formas de violencia y discriminación contra las mujeres, que a pesar del desarrollo de importantes marcos jurídicos que buscan atacar dichas formas de misoginia, "la ley tradicional del estatus se filtra en la ley moderna del contrato jurídico" (p. 138). En este sentido, concebimos que indagar las formas en las que la discapacidad es aprehendida en el discurso de docentes puede brindarnos algunas claves de lectura sobre el estatus de las personas funcionalmente diversas en el sistema educativo.

El texto que se desarrolla a continuación está dividido en cuatro apartados principales: en el primero se abordan aspectos teóricos relacionados con los modelos sobre la dis/capacidad y el capacitismo; seguidamente, se presenta el abordaje metodológico utilizado en la investigación; posteriormente, se incluye el apartado en el que se desarrolla el análisis de los resultados, el cual está subdividido en varias secciones correspondientes a los ejes o nodos discursivos identificados y, finalmente, se presenta un apartado de conclusiones y reflexiones finales del proceso de investigación.

\section{Referente teórico}

\subsection{Discursos sobre la dis/capacidad y la visión crítica del capacitismo}

Existen distintas concepciones para comprender el fenómeno denominado discapacidad. A lo largo de la historia, han existido formas bastante disímiles de dar sentido a las diferencias a nivel de la corporalidad y de las capacidades asociadas a los cuerpos. Para Palacios (2008), esa amplia variedad de localizaciones se puede clasificar en tres grandes modelos: Los modelos de la prescindencia, rehabilitador y social. A continuación, se describen dichos modelos retomando los lineamientos brindados por esta autora, estableciendo un nexo entre el modelo social de la discapacidad y el planteamiento de la diversidad funcional. 
Posteriormente, se abordará la noción de capacitismo (Nario-Redmond, 2020), que conlleva a una visión genealógica de la noción de discapacidad y que destaca el prejuicio y discriminación que experimentan las personas con discapacidad. Tomando en cuenta el interés que reviste esta última noción en el presente texto, a partir de este momento de la exposición se utilizará la forma de escritura de "dis/capacidad", cada vez que quiera destacarse dicho posicionamiento teórico.

De acuerdo con Palacios (2008), el modelo de la prescindencia, es aquel en el que se supone que las causas que dan origen a la discapacidad tienen un motivo religioso y en el que las personas con discapacidad son consideradas como innecesarias por diferentes razones. Como consecuencia de ello, la apuesta de la sociedad consistirá en prescindir de las personas con discapacidad, ya sea a través de la aplicación de políticas eugenésicas o excluyéndolas del espacio social. Este modelo deriva en una lógica de dependencia y sometimiento en el que las personas son tratadas como objeto de caridad y sujetos de asistencia.

Por su parte, siguiendo nuevamente a Palacios (2008), el modelo rehabilitador es aquel que considera que las causas que originan la discapacidad son científicas y que atañen a limitaciones individuales de las personas. De esta forma, las personas con discapacidad no son consideradas inútiles o innecesarias per se, puesto que tendrían la posibilidad de ser rehabilitadas. En este sentido, el fin principal al que apunta este modelo es normalizar a las personas con discapacidad, aunque ello implique el ocultamiento de la diferencia que la misma discapacidad representa.

Si bien es cierto que ambos modelos han ido perdiendo fuelle, persisten todavía algunas de sus secuelas, como las lógicas lastimeras con las que se suelen abordar diversas variaciones funcionales o las alternativas brindas a la exclusión de personas con discapacidad de determinados ámbitos sociales. Un claro ejemplo en este sentido es el discurso caritativo sobre el cual se basa la incorporación de personas con discapacidad al mercado laboral (Van Aswegen, 2019). El trabajo se convierte, así, en medio de rehabilitación social frente a una condición de exclusión naturalizada.

Finalmente, está el modelo denominado social, el cual, como señala Palacios (2008), es aquel que "considera que las causas que originan la discapacidad no son ni religiosas, ni científicas, sino que son, en gran medida, sociales" (p. 26). Como señala la autora, este modelo se relaciona con la asunción de ciertos valores intrínsecos a los derechos humanos, y parte de la premisa de que la discapacidad es en gran medida una construcción y un modo de 
opresión social, y el resultado de una sociedad que no considera ni tiene presente a las personas con discapacidad.

La noción de normalidad resulta un aspecto central en este sentido, puesto que abre la puerta a diversas vías analíticas, así, por ejemplo, siguiendo a Mareño y Masuero (2010), al concebir los procesos de "discapacitación social" es posible comprender que la discapacidad: No es una condición o atributo personal, sino una creación humana que todos los actores sociales producimos día a día, a partir de nuestras actitudes [capacitistas], decisiones, acciones y omisiones en un contexto pensado, diseñado y construido según parámetros convencionales y estereotipos de normalidad biomética (p. 103)

Como indican Dirth y Branscombe (2018), el modelo social de la discapacidad replantea la discapacidad como una cuestión social y no solo como una preocupación individual. Al hacerlo, el modelo social alienta a que se reconsidere la forma en que las diferencias de discapacidad no son intrínsecamente patológicas, sino que están mal situadas en un mundo construido para una franja demasiado estrecha de personas. Este modelo es por lo tanto una forma de trascender las visiones más conservadoras con las cuales se han tratado de explicar las divergencias corporales y la funcionalidad asociada a estas.

En una línea de continuidad como el modelo social, está lo que podríamos llamar el submodelo o la perspectiva de la diversidad funcionaR. Mediante la enunciación de dicho concepto se alude:

A una realidad en la que la persona funciona de manera diferente o diversa de la mayoría de la sociedad. Este término considera la diferencia de la persona y la falta de respeto de las mayorías, que en sus procesos constructivos no tienen en cuenta esa diversidad funcional. (Palacios y Romañach, 2006, p. 108).

Existen diversas discusiones sobre la pertinencia de utilizar esta noción, dado que, en un sentido estricto, todas las personas somos en alguna medida funcionalmente diversas. De igual forma, existen posicionamientos que claramente antagonizan con el uso de esta conceptualización, tal es el caso del Comité Español de Representantes de Personas con Discapacidad (CERMI) ${ }^{3}$, para quienes su uso "no describe la realidad sino que resulta confuso

\footnotetext{
2 Sobre este aspecto se puede encontrar una elaboración más amplia en: Fernández, D. (2016). Educación de personas con diversidad funcional. Algunos apuntes históricos. Revista Actualidades Investigativas en Educación. 3 Resulta particularmente relevante destacar aquí el planteamiento de dicho Comité español, puesto que la discusión sobre el concepto de diversidad funcional se generó en primera instancia en España.
} 
e incluso en ocasiones pretende ocultar esa realidad, atacando el enfoque inclusivo y de defensa de derechos" (CERMI, 2007, p. 5).

Ahora bien, en tanto que "la diversidad funcional logra abarcar un amplio espectro de dimensiones de la experiencia humana, adquiere valor la motivación y la propuesta de comenzar a tomarla realmente en serio en el ámbito epistémico-político" (Toboso, 2018, p. 801). Desde nuestra perspectiva, tomar en serio la propuesta de la diversidad funcional en el ámbito epistémico - político implica, más allá de abogar por su uso como categoría descriptiva, acoger las consecuencias teóricas de su formulación. Es, justamente, en este sentido que consideramos necesario traer a colación dicha noción, puesto que el hecho de que la sociedad confiera un carácter negativo a un conjunto determinado de características tanto físicas como mentales es la base para el establecimiento de distintas formas de discriminación, y en esa medida consideramos importante reconocer que dicha crítica puede nutrirse de distintos flancos.

Como un último elemento para tener en cuenta en relación con el objeto de estudio aquí presentado, el discurso sobre la discapacidad, tenemos la noción de capacitismo, que vendría a ser la traducción de la palabra "ableism" en inglés, lengua en la cual se han generado las principales reflexiones teóricas en torno a dicho concepto. La idea de capacitismo pone en cuestión una concepción bastante común de la discapacidad, según la cual, esta no sería una consecuencia más de la diversidad humana, sino un error frente a la norma social de lo que se concibe como un cuerpo capacitado. De acuerdo con Reynolds (2017), la configuración de la lógica capacitsta responde al menos a las siguientes cuatro condiciones:

(1) La discapacidad es conceptualizada como la falta o privación de un bien natural.

(2) La privación de bienes potencialmente naturales se considera un daño.

(3) Se entiende que el daño causa o es una forma de dolor y sufrimiento.

(4) Dado 1-3, en su versión débil, la discapacidad comprende el sufrimiento que estaría vinculado con la muerte. Mientras que en la versión fuerte, dicho dolor es la causa que conduciría a la muerte.

La noción de capacitismo, no solo cuestiona la idea de la dis/capacidad como anormalidad, sino que también conlleva una visión crítica respecto a la supuesta normalidad que sustenta el canon capacitista. Aunque resulta complejo, abordar la arista no problematizada de la "normalidad", constituye una tarea necesaria, pues como señala Scuro (2018), aunque la diferenciación corporal y la pluralidad experiencial de las narraciones han 
ido adquiriendo cierto capital cultural, como en el caso del ámbito educativo, este capital ampliado no desafía necesariamente los marcos capacitistas más dominantes y subrepticios, y por ello resulta imprescindible analizar críticamente ese costado poco discutido de lo normal, si es que se quiere alcanzar transformaciones más profundas en la sociedad.

\subsection{Cuerpos diversos en el sistema educativo}

Continuando la reflexión sobre las formas de asir la dis/capacidad es importante tener en cuenta su estrecha relación con el ámbito educativo. Davis (2000) señala que la discapacidad es una relación socialmente determinada con el cuerpo, que fue relativamente organizada en los siglos XVIII y XIX, y propulsada por factores económicos y sociales como parte de un proyecto más general de control y regulación de este. El sistema educativo formal, al conformar un aspecto central en el engranaje de la sociedad contemporánea, ha constituido un agente activo en ese proceso de control y regulación del cuerpo, que opera en mayor o menor medida en determinados aspectos de la subjetividad.

En esta línea, resulta relevante el planteamiento de McRuer (2006), quien acuña la noción de crip theory ${ }^{4}$. Para este autor, existe una interrelación de factores que permiten entender el lugar de los cuerpos funcionalmente diversos en el espacio cultural. De esta forma, como señala el autor, existen una demanda social de producir estudiantes que se acoplen a lógicas de rendimiento estandarizado, de una forma que no dista mucho del encargo social asociado al capacitismo corporal y a la heterosexualidad, se trata en todos los casos de un conjunto de demandas que imponen ajustarse a las exigencias del status quo.

Nuevamente aquí, la noción de normalidad es un aspecto clave para pensar el sistema educativo en relación con el sesgo capacitista. Siguiendo a Dubet (2005), las instituciones educativas no son realmente entidades imparciales, por cuanto las decisiones de orientación del estudiantado no están extensas ni de prejuicios ni de los pesos sociales que se atribuyen, por ejemplo, al género, la raza o la clase, a pesar de que la abrumadora mayoría de las personas docentes no son «sexistas», «racistas» «clasistas». A lo anterior se puede añadir que lo mismo acontece con el capacitismo, el cual tiene un importante peso en la forma en la que se organiza el sistema educativo en su conjunto, a pesar de que las personas que lo conforman no necesariamente estarían en contra de la diversidad funcional.

\footnotetext{
${ }^{4}$ Esta formación nominativa suele ser traducida como "teoría lisiada" o "teoría tullida", esta última especialmente en España. Es importante tener en cuenta que crip, al igual como sucede con el término queer, constituyen ambos vituperios en inglés y por lo tanto llevan una importante carga de injuria.
} 
Una premisa clave en el presente texto es que, de manera estrechamente relacionada con la cuestión de las condiciones psíquicas de la subjetividad, está la preocupación acerca de los límites corpóreos de la subjetividad. Para Grosz (1996), la manera en que el cuerpo es vivido y representado, así como los acercamientos y efectos de la corporalidad del sujeto en su identidad parecen aspectos cruciales a pesar de ser usualmente factores desestimados en cualquier explicación sobre el sujeto.

Como señala Erevelles (2011), las ideologías presentes en el currículo escolar sirven para construir sin querer ciertas subjetividades del estudiantado como desviadas, perturbadoras y peligrosas, justificando así su exclusión, razón por la cual resulta necesario preguntarse ¿en qué medida los valores de heteronormatividad, capacitismo, masculinidad y blancura se funden en la constitución cultural de la escuela? La apuesta que se plantea aquí, consiste en tratar de leer entre líneas los discursos del profesorado sobre la discapacidad, con miras a problematizar las formas de subjetividad que estos movilizan.

\section{Metodología}

\subsection{Enfoque}

El presente artículo se desarrolla mediante una metodología cualitativa, de carácter transeccional, con un alcance descriptivo y se inscribe dentro del ámbito de los estudios discursivos. La investigación se desarrolla específicamente desde la perspectiva del análisis crítico del discurso. La investigación de la cual deriva este texto se desarrolló entre el año 2017 y el año 2019.

\subsection{Unidades de análisis}

En esta investigación se entrevistaron a 10 docentes de dos colegios del área metropolitana, los cuales reciben mayoritariamente a una población de bajos recursos o de un nivel económico medio. El contacto con el profesorado se realizó a través del Departamento de Salud y Ambiente del Ministerio de Educación Pública de Costa Rica, el cual es el ente encargado de los temas de diversidad en un amplio espectro en dicha institución. Para el desarrollo de las entrevistas, las personas entrevistadas firmaron un consentimiento informado en el cual se garantiza la confidencialidad y anonimato de su participación. Por su parte, el proyecto que dio lugar al presente texto contó con la aprobación del Comité Ético Científico de la Universidad de Costa Rica (CEC-UCR). 


\subsection{Técnicas de recolección}

Como instrumento de recolección de la información se utilizaron entrevistas semiestructuradas. En dichas entrevistas se abordaron los siguientes tópicos: a) conocimiento general en torno a la diversidad, b) conocimiento sobre el trabajo con estudiantes con discapacidad, c) acercamiento a la discapacidad y los derechos de dicha población, d) experiencias específicas relacionadas a la labor docente con estudiantes con discapacidad.

\subsection{Procesamiento de análisis}

En lo que se refiere a la sistematización de la información, en primera instancia se realizaron grabaciones en audio que tuvieron una duración aproximada de entre 20 y 30 minutos, las cuales fueron transcritas según disposiciones de transcripción para corpus orales (Calsamiglia y Tusón, 2007). Mediante el programa Atlas-ti 8, se hizo una selección de fragmentos o citas textuales, las cuales posteriormente fueron clasificadas en códigos que hicieran referencia a los ejes temáticos de las preguntas relacionadas con el tópico de la discapacidad. En este sentido, el proceso de codificación fue de índole esencialmente deductiva.

Se seleccionaron fragmentos textuales más reiterativos en relación con el tema de la discapacidad, y posteriormente se utilizó la propuesta metodológica desarrollada por Vergara (2018), según la cual los fragmentos son descompuestos en proposiciones e implicaciones, entendiendo que las proposiciones representan el contenido semántico explícito de los textos, al tiempo que las implicaciones constituyen el contenido sugerido que se deriva del texto. En el Tabla 1 se muestra un ejemplo del procedimiento llevado a cabo con los diversos fragmentos textuales: 
Tabla 1. Ejemplo de procedimientos de análisis textual para entrevistas a docentes sobre el abordaje de la discapacidad.

\begin{tabular}{|c|c|c|}
\hline FRAGMENTO & PROPOSICIONES & IMPLICACIONES \\
\hline $\begin{array}{ll}\text { el tema } & \text { de } \\
\text { diversidad } & \text { de } \\
\text { discapacidad ya } \\
\text { es mucho más }\end{array}$ & $\begin{array}{l}\text { P1: el tema de la } \\
\text { discapacidad ya es } \\
\text { muchorrós } \\
\text { aceptado. }\end{array}$ & $\begin{array}{l}\text { I1: El tema de la discapacidad antes era menos } \\
\text { aceptado. } \\
\text { I2: El hablante hace referencia al colegio. }\end{array}$ \\
\hline $\begin{array}{l}\text { aceptado (...), por } \\
\text { ejemplo aquí } \\
\text { tenemos un chico } \\
\text { que tiene una } \\
\text { manita un tanto }\end{array}$ & $\begin{array}{l}\text { P2: aquí tenemos } \\
\text { un chico que tiene } \\
\text { una manita un tanto } \\
\text { deforme. }\end{array}$ & $\begin{array}{l}\text { 13: Hay otros y otras estudiantes sin deformidades. } \\
\text { 14: El hablante hace referencia al colegio. } \\
\text { 15: La forma del brazo del estudiante es una } \\
\text { característica diferenciadora. } \\
\text { 16: Hay cuerpos marcados visualmente y otros que no. }\end{array}$ \\
\hline $\begin{array}{l}\text { deforme, tiene } \\
\text { novia, para la } \\
\text { novia ni siquiera le }\end{array}$ & P3: tiene novia & $\begin{array}{l}\text { 17: Un estudiante con discapacidad puede tener novia. } \\
\text { I8: El que un estudiante con discapacidad tenga pareja } \\
\text { resulta poco habitual. }\end{array}$ \\
\hline $\begin{array}{l}\text { ve el: el detalle } \\
\text { ves, eso está, y } \\
\text { yo lo veo de la } \\
\text { mano y él va de la }\end{array}$ & $\begin{array}{l}\text { P4: para la novia ni } \\
\text { siquiera le ve el } \\
\text { detalle }\end{array}$ & $\begin{array}{l}\text { 19: La discapacidad del estudiante constituye una } \\
\text { característica ("detalle") notorio. } \\
\text { 110: La novia del estudiante con discapacidad no presta } \\
\text { atención a su discapacidad a pesar de su notoriedad. }\end{array}$ \\
\hline $\begin{array}{l}\text { mano buena de la } \\
\text { novia y la otra, la } \\
\text { manita corta que } \\
\text { tiene normal y } \\
\text { ella lo ve y lo ve } \\
\text { guapo y lo ve }\end{array}$ & $\begin{array}{l}\text { P5: yo lo veo de la } \\
\text { mano y él va de la } \\
\text { mano buena de la } \\
\text { novia }\end{array}$ & $\begin{array}{l}\text { I11: A pesar de la discapacidad del estudiante, este } \\
\text { anda de la mano con su pareja. } \\
\text { I12: Una de las manos del estudiante con discapacidad } \\
\text { es/está mala. } \\
\text { I13: Una de las manos del estudiante con discapacidad } \\
\text { es/está buena. }\end{array}$ \\
\hline $\begin{array}{l}\text { lindo y anda de la } \\
\text { mano con su } \\
\text { muchacho si para } \\
\text { ella fuera una: }\end{array}$ & $\begin{array}{l}\text { P6: y la otra, la } \\
\text { manita corta que } \\
\text { tiene, normal }\end{array}$ & $\begin{array}{l}\text { I14: A pesar de las características de la mano del } \\
\text { estudiante, su lugar en la interacción es normal. } \\
\text { I15: La mano corta del estudiante con discapacidad no } \\
\text { interfiere en la interacción con su novia. }\end{array}$ \\
\hline $\begin{array}{lr}\text { discapacidad, no } \\
\text { sería su novia } \\
\text { entonces, } \\
\text { volvemos a lo } \\
\text { mismo } & \text { el } \\
\text { concepto } & \text { de }\end{array}$ & $\begin{array}{l}\text { P7: ella lo ve y lo ve } \\
\text { guapo y lo ve lindo }\end{array}$ & $\begin{array}{l}\text { I16: El estudiante con discapacidad nota que su pareja } \\
\text { tiene una discapacidad. } \\
\text { I17: La novia del estudiante con discapacidad lo ve } \\
\text { lindo y guapo. } \\
\text { I18: La novia del estudiante con discapacidad lo ve } \\
\text { lindo y guapo a pesar de su discapacidad. }\end{array}$ \\
\hline $\begin{array}{l}\text { limitaciones y } \\
\text { discapacidad es } \\
\text { más del adulto } \\
\text { que del niño o el }\end{array}$ & $\begin{array}{l}\text { P8: y anda de la } \\
\text { mano con su } \\
\text { muchacho }\end{array}$ & $\begin{array}{l}\text { 119. Un estudiante con discapacidad puede ser } \\
\text { considerado "el muchacho" de una estudiante. } \\
\text { I20. Una estudiante a pesar de no tener discapacidad, } \\
\text { anda de la mano con un estudiante que sí tiene. }\end{array}$ \\
\hline $\begin{array}{l}\text { muchacho } \\
\text { (Prof. 1) }\end{array}$ & $\begin{array}{lr}\text { P9: si para ella } \\
\text { fuera } & \text { una } \\
\text { discapacidad, } & \text { no } \\
\text { sería su novia } & \end{array}$ & $\begin{array}{l}\text { 121. Considerar algo como una discapacidad es razón } \\
\text { para no ser novia de alguien. } \\
\text { I22. La estudiante no considera como una } \\
\text { discapacidad las características de su pareja y por eso } \\
\text { es su novia. }\end{array}$ \\
\hline & $\begin{array}{l}\text { P10: el concepto de } \\
\text { limitaciones y } \\
\text { discapacidad es } \\
\text { más del adulto que } \\
\text { del niño o el } \\
\text { muchacho }\end{array}$ & $\begin{array}{l}\text { I23. El uso de los conceptos de "limitaciones" y } \\
\text { "discapacidad" son solo atribuibles a personas adultas. } \\
\text { I24. El uso de los conceptos de "limitaciones" y } \\
\text { "discapacidad" no son atribuibles a personas no } \\
\text { adultas. } \\
\text { I25. Estar en una relación con un o una estudiante con } \\
\text { determinada característica física significa que no se } \\
\text { tiene un concepto de limitación y discapacidad. }\end{array}$ \\
\hline
\end{tabular}

Fuente: Elaboración propia basada en el modelo utilizado por Vergara (2018). 
La elaboración de implicaciones se realizó en primera instancia con la participación del equipo de asistentes con que contó el proyecto, con el cual se discutieron el sentido de las afirmaciones. Posteriormente, las implicaciones derivadas de los fragmentos textuales utilizados en este artículo fueron analizadas con la colaboración de dos profesionales en psicología. En el apartado de resultados que se presenta a continuación, se muestra la síntesis de dicho proceso de análisis textual.

\section{Resultados}

En concordancia con los planteado por Becker (2010), resulta "muy interesante el hecho de que la mayor parte de la investigación científica y la especulación sobre la desviación se ocupen más de las personas que rompen las normas que de quienes las producen o aplican" (p. 189). Dado que el cuerpo docente tiene el rol de detentor de la norma en el espacio colegial en general y en el espacio áulico en particular, concebimos que, en lo que respecta a la diversidad funcional, este rol es aún más relevante, puesto que los apoyos y facilidades de acceso que brinde el personal docente serán determinantes para el decurso del desarrollo académico e interaccional del estudiantado.

En este sentido, a partir de las entrevistas llevadas a cabo con el profesorado, fue posible identificar distintos discursos en torno a la discapacidad. Los tres principales discursos identificados en el corpus son los siguientes:

(1)Discurso basado en la dicotomía normalidad / anormalidad:

-La discapacidad en medio del discurso médico y el discurso educativo

-Discurso de la discapacidad como deficiencia

(2)Discurso de la discapacidad como una "carga" en el sistema educativo

(3) Discurso crítico sobre la diversidad funcional

\subsection{Discurso basado en la dicotomía normalidad / anormalidad}

Como se indicó en apartados anteriores, este artículo versa sobre el análisis del discurso de docentes de secundaria en torno a la discapacidad. Visto desde una lógica capacitista, la discapacidad representa una alteración al orden establecido, constituye una desviación de los parámetros de funcionalidad que legitiman la posibilidad de integrar el orden social. Conviene plantearse cómo se zanja la discrepancia entre lo que se concibe como la funcionalidad "desviada" y "encarrilada", o, en otros términos, qué configura la oposición normalidad versus 
anormalidad en el caso de la dis/capacidad. A continuación, se destacarán dos discursos en los que se asienta esta oposición.

\subsubsection{La dis/capacidad en medio del discurso médico y el discurso educativo}

Dentro de la dicotomía normalidad / anormalidad, uno de los discursos que muestra una importante presencia en el caso de las personas entrevistadas se refiere al hecho de entender la discapacidad como algo que necesariamente se padece, y cuyo principal correlato es el saber médico. En torno al par medicina - educación, conviene recordar que la retórica médica sobre la "discapacidad", así como el modelo educativo prusiano (Gatto, 2001), proliferaron en el seno de la razón centro europea de los siglos XVIII y XIX, razón por la cual no es de extrañar que existan parangones entre ambas esferas. La siguiente cita brinda algunos indicios en esta línea:

(1)Tal vez la, esas situaciones o capacidades especiales que tienen algunos, o que tenemos porque en algún momento uno pueda ser que tenga alguna: este, situación también eh, que, que, que tenga que adaptar digamos alguna capacidad especial verdad, o que hoy no la tenga y mañana por un accidente 0 , o lo que sea pueda que la tenga o por una enfermedad verdad (Prof. 9)

Un elemento muy relevante de esta intervención es que al inicio se plantea tomando en consideración la noción de "capacidad". Pensar la dis/capacidad en estos términos abre la posibilidad de pensar en el carácter contingente de las características del entorno, lo que, correspondientemente, permite pensar de distintas maneras la idea de "adaptación". La determinación de si se es "capaz de algo" estará dada en función de las propias potencialidades y de las características del contexto en que estas tienen lugar.

Ahora bien, el resto de implicaciones que derivan del fragmento apuntan a que las contingencias asociadas a la condición de (in)capacidad están fundamentalmente referidas al sujeto al que se identifica como "discapacitado". En otros términos, el agente de las contingencias es la persona en sí y no el contexto social en que se encuentra inmerso. Esta adscripción se puede leer fundamentalmente en dos direcciones, ya sea como resultado de un accidente o una enfermedad. De esta forma, aunque la persona aludida no tenga injerencia en la causalidad de ambos fenómenos, al convertirse en la portadora de su efecto, se convierte en la única responsable de su afrontamiento. 
Por su parte, el emparentamiento con las ideas de "accidente" y "enfermedad" conllevan una valoración negativa de la dis/capacidad, en la medida en que se refieren a un acontecimiento y condición indeseadas respectivamente. Un aspecto no menos importante, es que, a partir de lo señalado por parte de la entrevistada, tener un acercamiento empático con las personas funcionalmente diversas no sería un fin en sí mismo, puesto que en esencia estaría referido a la posibilidad de encontrarse en un futuro en una condición similar a la de estas.

En el discurso del personal docente, el saber médico se enuncia de diferentes maneras, una de ellas se refiere al uso de recursos léxicos, como se muestra en el siguiente fragmento:

(2)Bueno no soy muy conocedora del tema [discapacidad] básicamente las, lo que va recibiendo uno en, los años de formación que, en los últimos años en relación con el tema de adecuaciones, digamos que nos hemos capacitado un poquito más, en algunas sintomatologías verdad y en algunas cosas que uno de repente no sabe qué cómo abordarlos (Prof. 10)

En primera instancia, es necesario situar el sentido general de esta afirmación. Al inicio, mediante el señalamiento en que la docente indica: "no soy muy conocedora del tema", la diversidad funcional entra a formar parte de una instancia discursiva singular. Conlleva, como señala Foucault (1999), un efecto de enrarecimiento del discurso, en adelante se tratará de una cuestión que no atañe a la generalidad del cuerpo docente, sino que compete a algunas personas avezadas en la materia, aquellas a las que se puede calificar como "conocedoras".

Una vez concebidas las variaciones de la funcionalidad como un asunto que se ubica en una determinada relación de exterioridad con el quehacer docente habitual, el saber médico pasa a tener un valor preponderante. Hablar de "sintomatología", indefectiblemente refiere a manifestaciones que revelan determinados padecimientos o enfermedades. Argüimos en este sentido que esta operación conlleva inscribir la dis/capacidad en un orden médico a lo interno del ámbito educativo.

Otro aspecto que entra a jugar un rol importante en el discurso sobre la diversidad funcional se refiere a la valoración de la magnitud de aquello que se concibe como una discapacidad:

(3)Si usted me habla a mí de la palabra discapacidad, ya, estamos hablando ya de, siento yo ya de un problema eh, de aprendizaje más significativo, ya tirándolo a ese lado, digamos yo no veo, por ejemplo, un síndrome digamos este de atención, déficit de 
atención yo no veo un déficit atencional ni, una dislexia ni, nada de esas cosas las veo como una discapacidad (Prof. 3)

En este fragmento se busca establecer a qué remite y a qué no la palabra "discapacidad". En primera instancia, se específica aquello que no cabría dentro de dicha categoría, a saber, determinados tipos de "síndromes" como el llamado déficit atencional o la dislexia. Por su parte, se señala que hablar de discapacidad, significa hablar de un problema que estaría más allá de las dificultades asociadas a las condiciones antes referidas (se trata "ya de un problema de aprendizaje más significativo").

Esta intervención, a pesar de no ser suficientemente explícita respecto a la definición de qué sería un problema "más significativo", plantea que el terreno por antonomasia de la discapacidad es el aprendizaje, por lo que el ámbito institucional tendría un rol preponderante a este respecto (se ahondará sobre este punto en los siguientes apartados). En nuestro criterio, las colindancias entre el discurso médico y un discurso educativo responden al hecho de que en ambos campos la cuestión de establecer y gestionar el estatuto de lo normal y lo anormal resultan aspectos centrales de su quehacer.

Dirimir dicho estatuto en el caso de la discapacidad conlleva lo que Van Aswegen (2019) describe, en relación con ámbito laboral, como un discurso profundamente discapacitante de la discapacidad, en el que las deficiencias y los impedimentos individuales pasan a un primer plano como las causas del desempleo. Llevado al plano educativo, la marca de la discapacidad como anormalidad recae igualmente en las características individuales del estudiantado, y partiendo del señalamiento de Baquero (2001), sería una importante base para la sospecha de educabilidad que se dirige a dicha población.

\subsubsection{Discurso de la discapacidad como deficiencia}

Otro acercamiento discursivo en el marco de la dicotomía normalidad / anormalidad, que si bien colinda con lógica medicalizada no se subsume en esta, y se refiere en este caso, al discurso de la deficiencia.

(4)De discapacidad la verdad es que si, eh conozco bastante la ley 7600 porque más bien había hecho una tesis de, sí, era en un centro educativo para personas con discapacidad entonces, si ahí sí completamente de acuerdo verdad, porque di obviamente todos merecemos la oportunidad, para salir adelante, porque no por el hecho de que una persona tenga una deficiencia significa que no pueda tener una inclusión en la sociedad entonces yo pienso que sí, que es muy importante en los centros educativos 
exista esa inclusión hacia las personas que posean algún tipo de capacidad especial (Prof. 5)

Como se muestra en este extracto, se trata de una hablante que tiene un determinado recorrido en lo que se refiere al tema de la discapacidad, puesto que por un lado manifiesta conocer la Ley 7600, que es el instrumento jurídico más importante con el que cuenta Costa Rica en lo que se refiere a los derechos asociados a la diversidad funcional y, por lado, señala haber realizado su trabajo final de graduación en un centro educativo para personas con discapacidad.

Por su parte, algunas de las proposiciones realizadas por la entrevistada evidencian planteamientos críticos que, en buena medida, se corresponden con su recorrido en la materia, como el hecho de abogar por la inclusión de personas funcionalmente diversas en la sociedad en general y en los centros educativos en específico. No obstante, la forma en la que se entiende la idea de "deficiencia" resulta desdecir los propósitos anteriormente esbozados, no tanto porque se hable de deficiencia en sí, sino por quién o qué se concibe que es el agente de la misma.

La "deficiencia" es algo que ciertas personas tienen, más específicamente, se trata de una condición de la cual son portadores algunos individuos, mientras que habría otros y otras que están exentos de ella. Así, haciendo uso de una construcción tautológica, diremos que la discapacidad es inherente a las personas a las que es inherente. En otros términos, la "deficiencia" es un producto de las personas y no del sistema educativo en el que estas se encuentran inmersas. Ante esto, la inclusión se convierte en un accionar que busca paliar la incompatibilidad de las características funcionales del estudiantado frente a las características del modelo formativo. De acuerdo con Nario-Redmond (2020), el prejuicio de la discapacidad es multifacético y está en evolución; es mucho más que una actitud negativa unitaria, puede expresarse de forma no intencionada o deliberada en creencias, emociones, conductas, así como en prácticas institucionales e individuales que dan lugar a un tratamiento perjudicial de las personas por motivos de discapacidad.

A pesar de que estas formas sutiles de prejuicio parecieran tener un calado hondo, al punto en que personas con sensibilización considerable en la materia tienden a reiterarla, lo cierto del caso es que el planteamiento de la docente muestra la apertura suficiente para generar otro tipo de acercamientos a la diversidad funcional en el sistema educativo. Caso 
contrario al que se muestra a continuación, en el que se consulta a un docente sobre cuáles han sido las principales dificultades en el trabajo con un estudiante con discapacidad:

(5) No trabaja, él no trabaja y entonces eh, muchas de las instituciones a este caso se le ha, se le ha visto aquí, desde, sétimo a lo que es noveno, se le ha visto como el pobrecito, inclusive la relación que hay familiar es igual y la mamá no quiere aceptar o no, no sé eh, que tiene un problema serio en, en su casa con este tipo de personas, el: el, desenvolvimiento de este muchacho en, en lo que es propiamente acá la institución es, bastante deficiente, bastante pero bastante deficiente él no ejecuta, proyectos, él no ejecuta, eh trabajo diario simple hace, hace las cosas ahí, apenas, apenas, está acostu...- malacostumbrado, a, que, se le debe, se le pasa, sin que se le evalúen contenidos (Prof. 4)

Aquí, aunque de igual forma la retórica de la deficiencia es el eje central de la elocución, tenemos un acercamiento muy diferente al de la anterior entrevistada. Un primer aspecto a tener en cuenta, es la generalización ("este tipo de personas") en la que incurre el hablante, al referirse a la situación familiar del estudiante que toma como ejemplo para describir las dificultades asociadas a su labor docente. El hacer alusión a una tipología de estudiante reafirma la poca permeabilidad a las diferencias del estudiantado en términos de funcionalidad, ergo: "hay un tipo de estudiantes que no funciona".

La queja del docente muestra además algo que, de acuerdo con Keys, McMahon y Viola (2014), suele suceder en relación con la presencia de personas con discapacidad en las instituciones educativas, y es el hecho dichos establecimientos muchas veces se han asemejado más a guarderías que a instituciones educativas, y como resultado de ello, muchos niños, niñas y jóvenes con discapacidad no han tenido la misma oportunidad de crecer y desarrollarse a través de la educación, la cual constituye una vía importante de empoderamiento para muchas personas.

Ahora bien, un aspecto muy importante a tener en cuenta es que la marca de la "deficiencia" está dada en relación a la valoración que hace el docente sobre el rendimiento del estudiante ("él no trabaja", "el desenvolvimiento de este muchacho es (...) bastante deficiente", "él no ejecuta proyectos", "él no ejecuta el trabajo diario simple", "hace las cosas ahí apenas"). Todas las proposiciones apuntan a algo que el estudiante no hace o que realiza de manera inadecuada, lo cual, por un lado, implica que no existe cuestionamiento alguno de si los trabajos y proyectos designados, o la forma en la que están formulados son los 
adecuados y, por otro lado, en consecuencia, la responsabilidad sobre el desempeño es atribuida de manera exclusiva al estudiante.

\subsection{Discurso de la discapacidad como una "carga" en el sistema educativo}

Uno de los discursos que está más presente por parte del profesorado sobre la dis/capacidad es que esta constituye una carga muy difícil de sobrellevar en el marco de su labor como docentes. En este apartado no se pretende dejar de lado el hecho de que, efectivamente, los equipos docentes cuentan en muchas ocasiones con considerables cargas laborales que dificultan desarrollar adecuadamente su labor profesional. No obstante, los análisis que proponemos en este apartado parten de la concepción de que el ejercicio de la docencia requiere de abordajes que propicien la integración del estudiantado en su multiplicidad de diferencias, entendiendo claro está, que dicha labor no compete exclusivamente al personal docente, sino que apela a una visión más bien estructural, de la cual el profesorado es el principal depositario.

En este sentido, concebimos que atender las particularidades de un discurso que construye la discapacidad como una "carga" constituye una vía relevante para dilucidar el funcionamiento de los engranajes de esa lógica estructural en la cual esta es aprehendida. En buena parte, tal funcionamiento macro, como señala McRuer (2019), estaría vinculado con la lógica del neoliberalismo como sistema económico imperante, aspecto que se ve claramente representado en las políticas de austeridad implementadas en muchos países, las cuales, por demás, tienen una injerencia directa en las condiciones de vida en general de las personas con dis/capacidad y en el acceso y permanencia de estas en los sistemas educativos en particular.

Dicho discurso aparece tematizado de distintas maneras en las intervenciones de las personas entrevistadas, así por ejemplo, un recurso que se suele esgrimir es enunciar la oposición de la labor que conlleva trabajar con estudiantes con y sin discapacidad. En esta línea, la siguiente cita constituye la respuesta que brinda una docente sobre cómo en su criterio debería ser el trabajo con personas funcionalmente diversas:

(6)Debería ser apoyo total, no es, no es secreto que a veces es difícil verdad, tal vez con adecuaciones ya significativas como a nivel, cognitivo que uno a veces, se dice se tiene que hacer esto yo sé y lo otro, pero ya en la clase pues ya es, es, es, es un poco difícil, por el tiempo tan, limitado que tiene uno, a veces con costos tiene uno para los 
chiquillos que no tienen ninguna discapacidad dice uno verdad, entonces hace difícil pero por lo menos en, en la anuencia a brindar el apoyo sí (Prof. 2)

En este extracto se oponen dos posicionamientos discursivos, por un lado, está la tendencia a reafirmar la importancia de brindar todo el apoyo que sea necesario para llevar a buen término el proceso formativo del estudiantado y por otro, se enuncian las dificultades para poner en práctica medidas que vayan en dicha dirección que, en este caso, alude a la implementación de adecuaciones significativas que se relacionan aquí con aspectos cognitivos, pero que podrían referir a otras características funcionales ${ }^{5}$.

En otros términos, se vislumbra un umbral a partir del cual resulta poco asequible atender las necesidades de estudiantiles, mismo que, en la segunda parte de la intervención, es atribuido al factor tiempo. Sobre este punto, se plantean a nuestro criterio dos importantes disyuntivas: ¿qué hacer con el tiempo que no hay?, y ¿para quién hacer con el poco tiempo que es posible invertir?

Entre las implicaciones que conlleva la proposición "a veces con costos tiene uno [tiempo] para los chiquillos que no tienen ninguna discapacidad", está que el poco tiempo disponible se dedica a las personas que no tienen ninguna discapacidad y que habría un tiempo todavía más exiguo para las personas que sí tienen una discapacidad. Lo anterior, a pesar de la anuencia manifestada a brindar apoyo, pinta un cuadro poco favorable para la diversidad funcional en el contexto colegial al que se hace referencia.

En concordancia con la idea de los "casos liminales" que se deja ver en el planteamiento anterior, en el siguiente extracto se enfatiza la manera en que son percibidas algunas de las dificultades que conlleva el trabajo con la dis/capacidad en un contexto colegial:

(7)A veces lo que pasa es que, eh que eso que son chicos con déficit atencional con hiperactividad o, o autistas o así y entonces tampoco, ellos como que, a los que son muy inquietos a veces ellos [profesores] no se los...- no se los soportan verdad (.) quieren ojalá se vayan de la institución...- en algunos casos...- no vamos a

\footnotetext{
${ }^{5} \mathrm{~A}$ tal propósito, tenemos la siguiente intervención que constituye la respuesta de una docente ante la pregunta de cuál considera que es el abordaje que se debe dar a estudiantes con discapacidad: "yo siento que tiene que ser totalmente individualizada, porque yo no puedo pretender que, por ejemplo por esa estudiante que tenía no vidente, di fuera al ritmo de los demás (Prof. 8)". Este planteamiento constituye una solución contraria en su accionar, pero homóloga en su resultado al que se plantea en (6). En un caso, se trata de la inacción como consecuencia de circunstancias complejas ("el tiempo tan limitado que tiene uno"), mientras que en el otro se trata de un accionar improbable ("[atención] totalmente individualizada”), ante circunstancias bastante similares.
} 
generalizar porque no podemos generalizar verdad tampoco pero diay hay casitos que uno dice ay dios (Prof. 10)

Esta última intervención plantea un contraste que conviene destacar, por cuanto, en una dirección se advierte de la importancia de no generalizar la posición de rechazo que manifiestan algunos docentes hacia las personas con discapacidad, mientras que en otra dirección se declara el porqué, hasta cierto punto, dichos posicionamientos resultan razonables o entendibles ("pero diay hay casitos que uno dice ay dios"). En este sentido, como señala Goodley (2014), pareciera que las personas con discapacidad que entran en el sistema educativo convencional contravienen los ideales de la educación.

Atender las diferencias que funcionalmente implican el déficit atencional o el autismo que por demás no pasan por ser "condiciones" excepcionales o atípicas en las instituciones educativas- pareciera suponer en este caso una dificultad mayor en el quehacer educativo. Más aún, frases como "no se los soportan" o "quieren ojalá se vayan de la institución", evocan la idea de que la diversidad funcional constituye en muchos casos un verdadero impase en la labor docente.

Las siguientes dos intervenciones retratan de manera palmaria esta imposibilidad a partir de los planteamientos de otros docentes:

(8) Cuesta que ellos, ellos desarrollen los proyectos ahora tengo un caso que, ${ }^{* * * * *}$ muy difícil, yo creo que, que, por ejemplo, para un colegio técnico, es SUMAMENTE difícil manejar ese tipo de personas (Prof. 4)

(9)Digamos, a veces los profesores...- en algunos casos verdad los profesores e, PREFIEREN, para no complicarse la vida, prefieren irlos pasando, en algunos casos (Prof. 9).

En la primera intervención se plantea la necesidad de establecer una separación entre estudiantes con y sin discapacidad, de tal forma que se excluya de los colegios que oferten una formación técnica al alumnado que presente algún nivel de funcionalidad divergente y no correspondiente a línea capacitista de la normalidad educativa. Se evoca, a su vez, la idea de una tipología de persona, enfatizando que se requiere de un "manejo" especial, que resultaría "sumamente difícil" de sostener para una institución con las características antes señaladas. 
Contrario a la primera intervención en la que prima una idea de hacer operar un accionar en el que se excluya de facto a las personas con discapacidad, la segunda tiene un efecto de exclusión más bien atenuado. Esta "solución" conlleva anular el compromiso de formación de un segmento de la población, lo que equivale a decir que en casos como el evocado aquí, la educación no reviste ningún interés, sino que, por el contrario, constituye un fardo que se lleva con gran dificultad.

De cualquier forma, argüimos que tanto en estos ejemplos como en otros que han sido presentados en esta sección, el discurso de la discapacidad pensada como una carga, está ligado estructuralmente a un ideal capacitista del cuerpo (McRuer, 2006), que retrata, por demás, una realidad poco halagüeña para las personas funcionalmente diversas en el contexto educativo.

\subsection{Discurso crítico sobre la diversidad funcional}

En este último apartado, destacamos dos intervenciones que, ya sea por el abordaje práctico al que aluden o, en razón de la visión que subyace a los criterios manifestados, coadyuvan en la configuración de espacios educativos que sean capaces de acoger la diversidad funcional en el ámbito educativo. La siguiente cita brinda algunos aspectos relevantes en este sentido:

(10) Tenemos casi todo tipo de adecuaciones, no significativas, las específicas, inclusive, eh, aquí en el aula trabajamos con personas con nivel funcional de tercer y cuarto grado y, les damos el acceso para, para que puedan cursar eh, aquí el colegio, como cualquier estudiante nada más con las adecuaciones respectivas y realmente en cuanto acceso aquí es excelente (Prof. 7).

Si se toma como criterio otras intervenciones planteadas anteriormente parecería que, en este caso, se está haciendo referencia a una institución muy diferente a algunas de las dos que formaron parte de este estudio, sin embargo, esto no es así, pues la docente entrevistada forma parte de uno de los dos colegios que se contemplaron en el estudio. De ello se pueden derivar varios aspectos, en primera instancia, que existe un marco institucional que provee una serie de alternativas que hacen viable la estancia de estudiantes con diversidad funcional en el sistema de educación regular ("como cualquier estudiante nada más con las adecuaciones respectivas"). 
Más allá de las disquisiciones que puedan darse en razón del criterio personal de cada docente, es importante atender al hecho de que, si bien es cierto que disponer de lineamientos y protocolos que permiten atender las diferencias del estudiantado en materia de funcionalidad resulta importante, dichos instrumentos se vuelven inocuos si no están acompañados de una verdadera sensibilización respecto al reconocimiento de las diferencias que implica justamente hablar de diversidad funcional. La siguiente intervención, muestra algunas claves en esta dirección:

(11) Mucho se dice que la discapacidad está en la mente de cada uno de nosotros (.) sin embargo no podemos negar que algunos tenemos diferentes potencialidades que es el caso de los chicos, yo no digo que hay una discapacidad, sino que hay una diferencia de talentos, algunos desarrollamos unos talentos que nos permiten económicamente, profesionalmente, salir y otros tenemos otros talentos que muchas veces, a la espera de una sociedad que sea económicamente, y profesionalmente se dejan de ver, es el caso de los chicos con necesidades educativas especiales, tienen unas capacidades que muchas veces, no surgen, por ideas propias de la comunidad, ideas propias de la familia, e inclusive la falta de credibilidad en muchas veces de los profesores que los tienen a cargo (Prof. 6)

Al tiempo que destaca que, en relación con la discapacidad existe un componente material que se refiere al hecho de que efectivamente existen diferencias a nivel funcional entre personas (mismas que inciden en distintos ámbitos como el económico o profesional), también se pone el acento sobre cómo influye la manera en la que son percibidas dichas diferencias ("está en la mente de cada uno de nosotros"). Esta visión crítica, o quizá deba decirse autocrítica, conlleva poner en tensión las bases mismas del estatus capacitista en el ámbito educativo, aspecto nada desdeñable, pues como indica Ferreira (2011):

No cabe plantear la inclusión escolar de las personas con discapacidad sin plantear una crítica, práctica a la propia institución escolar. Y esa crítica implica incorporar, como herramienta teórica e ideológica, una política del cuerpo que permita a las personas con discapacidad reapropiarse de su propia experiencia y vivencia concretas. (p. 17)

Finalmente, otro punto de reflexión que queremos destacar de la intervención de esta docente es que su planteamiento posiciona la diversidad funcional como un fenómeno en el que convergen distintas esferas (tales como la familia, la comunidad y el colegio -enmarcado 
en este caso en la figura docente-), y que todas ellas tienen mucha injerencia en el decurso de la vida de muchas personas ("en el caso de población infantil con necesidades educativas especiales, tienen unas capacidades que muchas veces no surgen"). En este sentido, si damos por cierto que dichas esferas, en general, y el colegio en particular tienen la capacidad de limitar las capacidades de las personas funcionalmente diversas es necesario asumir que tienen la posibilidad de generar el efecto contrario.

\section{Conclusiones}

Como parte de las limitaciones del presente artículo hay que destacar, en primera instancia, que, como toda investigación de carácter cualitativo, sus resultados no son generalizables a la totalidad de la población a la que se alude, en este caso a docentes de secundaria pertenecientes al sistema de educación pública costarricense. En esta dirección, otra carencia del estudio es la no inclusión de docentes pertenecientes al ámbito rural dado que las instituciones a las que pertenece el personal docente consultado forman parte del Gran Área Metropolitana. Tomando en cuenta estas consideraciones, los resultados aquí presentados aspiran a problematizar algunos núcleos discursivos respecto a lo que el profesorado entiende que es la discapacidad, aspecto que puede tanto servir como un insumo para el trabajo ulterior con docentes como para la elaboración de categorías analíticas para el desarrollo de investigación con un alcance más amplio.

En este sentido, una inquietud heurística que moviliza la elaboración de este texto es que, a pesar del importante desarrollo a nivel de legislación y políticas en materia de dis/capacidad, a nivel cultural persisten prejuicios que hacen que el proyecto de inclusión no se materialice de manera efectiva y en todos sus necesarios alcances en nuestra sociedad. En este sentido, y de acuerdo con Campbell (2009), aunque hay muchos debates en los estudios sobre la discapacidad, la mayoría de las personas estarían de acuerdo con el señalamiento de que las personas a las que se denomina "discapacitadas" experimentan diversos grados de subordinación y disminución de sus vidas a través de la discriminación económica, social, jurídica, religiosa y cultural.

Por los aspectos anteriormente señalados, el presente texto se abocó a analizar la visión del profesorado en torno a la diversidad funcional, con el objetivo de identificar algunos núcleos discursivos en torno a esta temática que nos permitiesen reflexionar más a fondo respecto a la forma en la que operan o no las transformaciones en el contrato social en el marco de las instituciones educativas. 
A partir de los análisis formulados, planearemos ahora algunas reflexiones finales, que como se indicó, respecto al enfoque metodológico del estudio, no buscan generalizar sus planteamientos ni tampoco pretenden obturar las reflexiones derivadas del proceso de investigación. Por el contrario, en la lógica que apunta el pensamiento situado (Haraway, 2004), se busca entender la forma que toma ese objeto de estudio que es la diversidad funcional desde el contexto y la subjetividad del personal docente que se entrevistó.

A partir del análisis de proposiciones e implicaciones, es posible afirmar que la dicotomía normalidad / anormalidad constituye un importante nodo discursivo que subtiende la visión sobre la diversidad funcional del personal docente que participó en la presente investigación. A nivel discursivo, esta oposición le permite al personal docente hacer inteligibles las diferencias funcionales del estudiantado. De esta forma, existen cuerpos normales y cuerpos anormales, y las capacidades asociadas a tales cuerpos serán concomitantes a dicha designación dicotómica.

En la medida en que las variaciones de la funcionalidad se ubican en una relación de exterioridad respecto a un quehacer docente que estaría pensado como "regular", "habitual" o "común”, el discurso médico adquiere un valor articulador, es decir, permite nombrar, describir y hasta cierto punto explicar eso que ocupa el lugar de "lo otro" en el escenario del colegio. Algo que comparte la lógica patologizante que encarna el discurso médico con la idea de la discapacidad como deficiencia, es que, en ambas configuraciones discursivas, tanto la idea de lo "deficiente" como de lo "patológico" constituyen una marca personal, de tal forma que el sistema educativo pareciera no tener parte alguna en la definición de ambas condiciones.

Por otro lado, tenemos el discurso de la discapacidad como una carga, que es quizá una de las representaciones que con mayor crudeza refleja un desajuste entre el ejercicio de la docencia y la diversidad funcional. Si bien es necesario tener en cuenta lo complejo del ejercicio de la docencia en contextos de una cada vez mayor desigualdad social y limitación de recursos, también es cierto que focalizar a las personas con necesidades educativas específicas como una carga excedente, refleja que existen personas cuyo valor socioeducativo es inferior al de otras.

Afortunadamente, es importante señalar que al lado de este tipo discursos, existen otros acercamientos que muestran una visión más crítica y empática respecto a la diversidad funcional en el ámbito educativo. Un aspecto a destacar de estos planteamientos es que los mismos hacen manifiesto el hecho de que, a pesar de que en el profesorado persisten discursos amparados en una lógica capacitista que redunda en la exclusión y la discriminación 
de las personas funcionalmente diversas, es posible generar otro tipo de relación con lo diverso y en esta medida, el colegio puede pasar de ser un espacio abocado meramente a tolerar la diversidad a uno que trabaje activamente por reivindicar su existencia.

\section{Referencias}

Asamblea Legislativa de Costa Rica. (1996). Ley No. 7600. Ley de Igualdad de Oportunidades para las Personas con Discapacidad. San José, Costa Rica: Diario Oficial La Gaceta.

Asamblea Legislativa de Costa Rica. (2008). Ley No. 8661: Convención sobre los Derechos de las Personas con Discapacidad y su Protocolo. San José, Costa Rica: Diario Oficial La Gaceta.

Asamblea Legislativa de Costa Rica. (2016). Ley para la Promoción de la Autonomía Personal de las Personas con Discapacidad N 9379. San José, Costa Rica: Diario Oficial La Gaceta.

Comité Español de Representantes de Personas con Discapacidad [CERMI]. (2017). Documento 1/2017 de Normas de Estilo y Expresión y Comunicación del CERMI estatal. Recuperado de http://www.sindromedown.net/wp-content/uploads/2017/10/NORMASDE-ESTILO-DEL-CERMI-ESTATAL.pdf

Baquero, Ricardo. (2001). La educabilidad bajo sospecha. Cuaderno de Pedagogía Rosario, 4(9), 71-85. Recuperado de https://www.researchgate.net/publication/328199034 La educabilidad bajo sospecha

Becker, Howard. (2010). Outsiders: Hacia una sociología de la desviación. Buenos Aires, Argentina: Siglo Veintiuno Editores.

Calsamiglia, Helena y Tusón, Amparo. (2007). Las cosas del decir: Manual de Análisis del discurso. Barcelona, España: Editorial Ariel.

Campbell, Fiona. (2009). Contours of Ableism: The Production of Disability and Abledness. New York, Estados Unidos: Palgrave McMillan.

Consejo Nacional de Rehabilitación y Educación Especial (CNREE). (2011). Política Nacional en Discapacidad 2011-2021. Heredia, Costa Rica: Consejo Nacional de Rehabilitación y Educación Especial.

Davis, Lennard. (2000). Enforcing Normalcy, Disability, Deadness and the Body. London: Verso.

Dirth, Thomas., y Branscombe, Nyla. (2018). Disability models affect disability policy Support through awareness of structural discrimination. Journal of Social Issues, 73(2), 413-442. doi: https://doi.org/10.1111/josi.12224

Dubet, François. (2005). La escuela de las oportunidades. ¿Qué es la escuela justa? Barcelona: Editorial Gedisa. 
Erevelles, Nirmala. (2011). "Coming our crip" in inclusive Education. Teachers College Review, 113(10), 2155-2185. Recuperado de https://eric.ed.gov/?id=EJ951090

Fernández, Daniel. (2016). Educación de personas con diversidad funcional. Algunos apuntes históricos. Revista Actualidades Investigativas en Educación, 16(3), 1-23. doi:http://dx.doi.org/10.15517/aie.v16i3.26064

Ferreira, Miguel. (2011). Discapacidad, globalidad y educación: ¿una nueva "política del cuerpo"? Revista Latinoamericana de Estudios sobre Cuerpos, Emociones y Sociedad,3(6), 6-19.

Recuperado de https://dialnet.unirioja.es/descarga/articulo/3716541.pdf

Foucault, Michel. (1999). El orden del discurso. Madrid, España: Tusquets Editores.

Gatto, John. (2001). Underground History of American Education. Nueva York, Estados Unidos: The Oxford Village Press.

González, Daniela., y Stang, Fernanda. (2014). Las personas con discapacidad en América Latina a 20 años del consenso de El Cairo: la necesidad de información para políticas y programas. En Comisión Económica para América Latina y el Caribe, Notas de Población, 99. (pp. de https://repositorio.cepal.org/bitstream/handle/11362/37634/np99067132 es.pdf;jses sionid=F2EA3F366777D590E375483500B19D52? sequence $=1$

Goodley, Dan. (2014). Dis/ability studies. Theorising disablism and ableism. London and New York, Estados Unidos: Routledge; Taylor \& Francis Group.

Grosz, Elizabeth. (1996). Intolerable Ambiguity: Freaks as/at the Limit. In: R. GarlandThomson(Ed.), Freaky: Cultural Spectacles of the Extraordinary Body (pp. 55-66). New York, Estados Unidos: New York University Press.

Haraway, Donna. (2004). The Haraway Reader. New York and London: Routlege.

Keys, Christopher., McMahon, Susan., y Viola, Judah. (2014). Including students with disabilities in urban public schools: community psychology theory and research. Journal of Prevention \& Intervention in the Community, 42(1),1-6. doi: https://doi.org/10.1080/10852352.2014.855025

Mareño, Mauricio., y Masuero, Fernanda. (2010). La discapacitación social del "diferente". Intersticios: Revista Sociológica de Pensamiento Crítico, 4(1),95-105. Recuperado de https://www.intersticios.es/article/view/4564

McRuer, Robert. (2006). Crip Theory. Cultural Signs of Queerness and Disability. New York, Estados Unidos: New York University Press.

McRuer, Robert. (2018). Crip Times: Disability, Globalization, and Resistance. New York, Estados Unidos: New York University Press. 
Meléndez, Raudin. (2018). Educación inclusiva y discapacidad en Costa Rica: una perspectiva desde las políticas públicas. Revista Actualidades Investigativas en Educación, 18(2),127. doi: https://doi.org/10.15517/aie.v18i2.33253

Nario-Redmond, Michelle. (2020). Ableism. The causes and consequences of disability prejudice. New York, Estados Unidos: Wiley Blackwell.

Palacios, Agustina. (2008). El modelo social de discapacidad: Orígenes, caracterización y plasmación en la Convención Internacional sobre los Derechos de las Personas con Discapacidad. Madrid, España: Ediciones Cinca.

Palacios, Agustina., y Romañach, Javier. (2006). El modelo de la diversidad. La bioética y los derechos humanos como herramientas para alcanzar la plena dignidad en la diversidad funcional. España: Ediciones Diversitas.

Quijano, Grettel. (2008). La inclusión: Un reto para el sistema educativo costarricense. Revista Educación, 32(1), 139-155.

Reynolds, Joel. (2017). "I'd rather be dead than disabled" - the ableist conflation and the meanings of disability. Review of Communication, 17(3),149-163. doi: https://doi.org/10.1080/15358593.2017.1331255

Scuro, Jennifer. (2018). Addressing Ableism: Philosophical Questions via Disability Studies. New York, Estados Unidos: Lexington Books.

Segato, Rita. (2003). Las estructuras elementales de la violencia: Ensayos sobre género entre antropología, el psicoanálisis y los derechos humanos. Buenos Aires, Argentina: Editorial Universidad Nacional de Quilmes.

Stang, Fernanda. (2011). Las personas con discapacidad en América Latina: del reconocimiento jurídico a la desigualdad real. Santiago, Chile: Centro Latinoamericano y Caribeño de Demografía. Recuperado de https://repositorio.cepal.org/bitstream/handle/11362/7135/S1100074 es.pdf?sequen $\underline{\mathrm{ce}}=1$

Toboso, Mario. (2018). Diversidad funcional: hacia un nuevo paradigma en los estudios y en las políticas sobre discapacidad. Política y Sociedad, 55(3), 783-804. doi: https://doi.org/10.5209/POSO.56717

Van Aswegen, Jennifer. (2019). Disabling discourses and charitable model of disability: Labour market activation for people with disabilities, Ireland - a critical policy analysis. Disability \& Society, 35(3), 1-25. doi: https://doi.org/10.1080/09687599.2019.1634519

Vergara, Adrián. (2018). El discurso sobre la discapacidad. Exclusión y auto-exclusión desde las familias de personas con discapacidad. Cadernos de Linguagem e Sociedade, 19(1), 209-231. doi: https://doi.org/10.26512/les.v19i1.10881 
Revista indizada en

sciplo

redalyc latindex

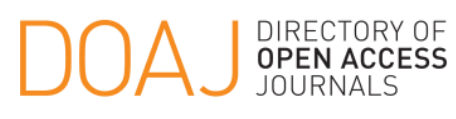

Distribuida en las bases de datos:

- Dialnet

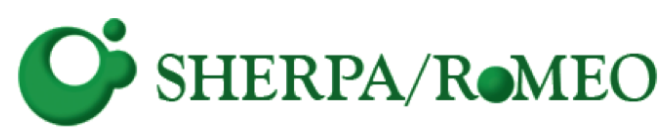

REDIB

Red Iberoamericana

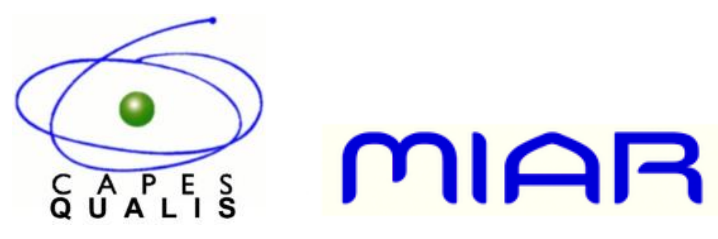

